\title{
Integrating baseline electrocardiography and myocardial perfusion imaging
}

\author{
Gregory S Thomas, MD, MPH, MASNC, ${ }^{\mathrm{a}, \mathrm{b}}$ and Arash Taghav, MD \\ a MemorialCare Heart \& Vascular Institute, Fountain Valley, CA \\ b Division of Cardiology, University of California Irvine, Orange, CA
}

Received Nov 12, 2020; accepted Nov 13, 2020

doi: 10.1007/s12350-020-02459-9

\section{See related article, pp. 810-821}

Drs. Khan, Arif, and Doukky provide us with insights from Cook County Health into the prognostic significance of ST and T wave abnormalities on the baseline ECG performed immediately prior to regadenoson myocardial perfusion imaging (MPI). ${ }^{1}$ Excluding patients with known CAD, LBBB, IVCD, and ventricularly paced rhythm, these investigators followed 6059 patients for a mean of 2.3 years post MPI for the occurrence of cardiac death and myocardial infarction (MACE). Using a low threshold for characterizing an ECG as abnormal-any ST segment below baseline or with an inverted T wave, 1912 (32\%) of patients had an abnormal baseline ECG. They divided these into those with ST and T waves abnormalities and those with isolated T wave inversion. Patients with ST and $\mathrm{T}$ wave abnormalities were further divided into those secondary to RBBB or left or right ventricular hypertrophy and those without RBBB and ventricular hypertrophy. The abnormalities in former group were termed secondary ST/ $\mathrm{T}$ wave abnormalities and the latter, primary ST abnormalities.

Figure 1 shows examples of each group.

They found MACE at 2.3 years to be statistically greater in those with secondary ECG abnormalities, $3.2 \%$, than in those with normal ECGs, $1.3 \%$. There was also a trend toward higher MACE in those with primary

Reprint requests: Gregory S Thomas, MD, MPH, MASNC, MemorialCare Heart \& Vascular Institute, Fountain Valley, CA; gthomas1@memorialcare.org

J Nucl Cardiol 2022;29:822-5.

$1071-3581 / \$ 34.00$

Copyright (c) 2021 American Society of Nuclear Cardiology.
ST abnormalities (2.0\%) but no difference in those with isolated $\mathrm{T}$ wave inversion $(1.2 \%)$.

The investigators chose to use automated MPI scoring using only quantitative scoring (Corridor 4DM) without visual over read or the incorporation of left ventricular (LV) ejection fraction (EF), wall motion or LV EF reserve (post-stress LV EF minus rest LV EF) into the determination of whether an MPI was abnormal or normal. Only those with summed stress scores (SSS) of $\geq 4$ were characterized as abnormal. Separately ischemia was determined to be present if the summed difference score was $\geq 2$. Using this scoring system, $18.5 \%$ of scans were abnormal and $18.6 \%$ were ischemic.

In the relatively short 2.3-year follow-up period, abnormal and ischemic MPI predicted MACE in patients with primary ST abnormalities and $\mathrm{T}$ wave inversion but not in those with secondary ST/T abnormalities. ${ }^{1}$ Events were relative few in each of these groups leading to a potential Type II error (too small a sample size, as the authors also note) in the group with secondary ST/T abnormalities.

\section{PREVIOUS STUDIES}

As one would expect, baseline ECG abnormalities in the general public without known CAD have also been found to predict adverse cardiac outcomes. Pedone et al. reviewed a number of these studies in their 2011 paper. ${ }^{2}$ Such studies include those of Daviglus et al. who found non-specific ST and T wave abnormalities to predict MI and cardiac and all-cause mortality in 1673 middle-aged men. ${ }^{3}$ Deeker et al. found ST depression to predict cardiac death and MI in middle-aged and elderly men. ${ }^{4}$ Auer et al. found an abnormal ECG to predict a composite of cardiac death, MI, coronary revascularization, and hospitalization for angina in 2192 men and women 70 to 79 years of age. ${ }^{5}$ 
A

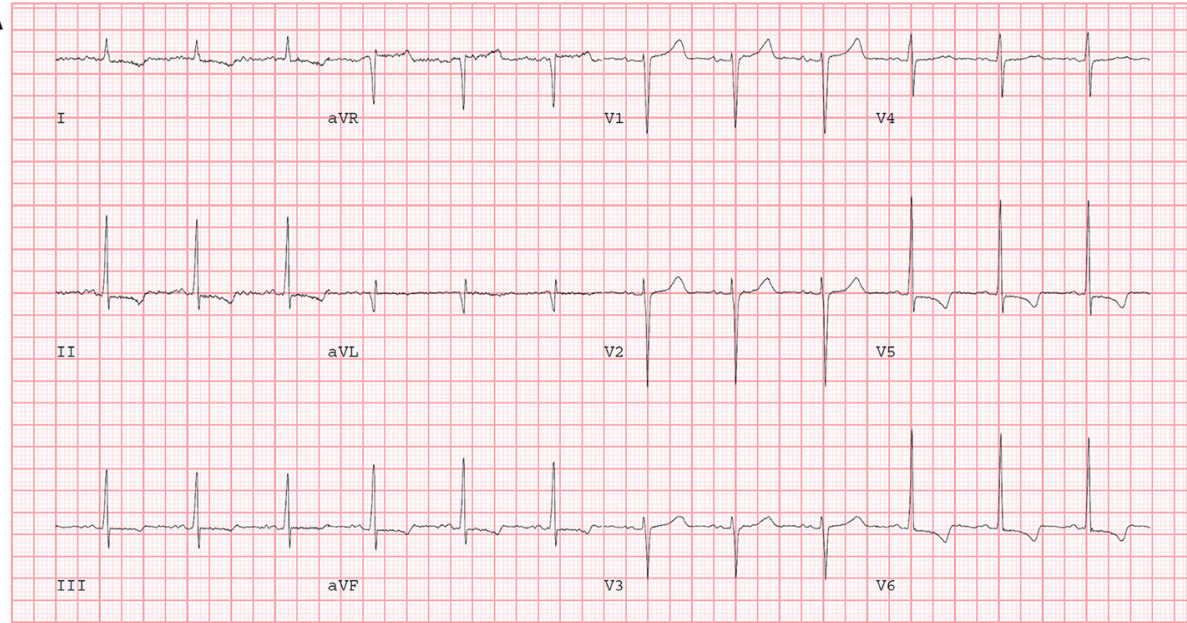

B

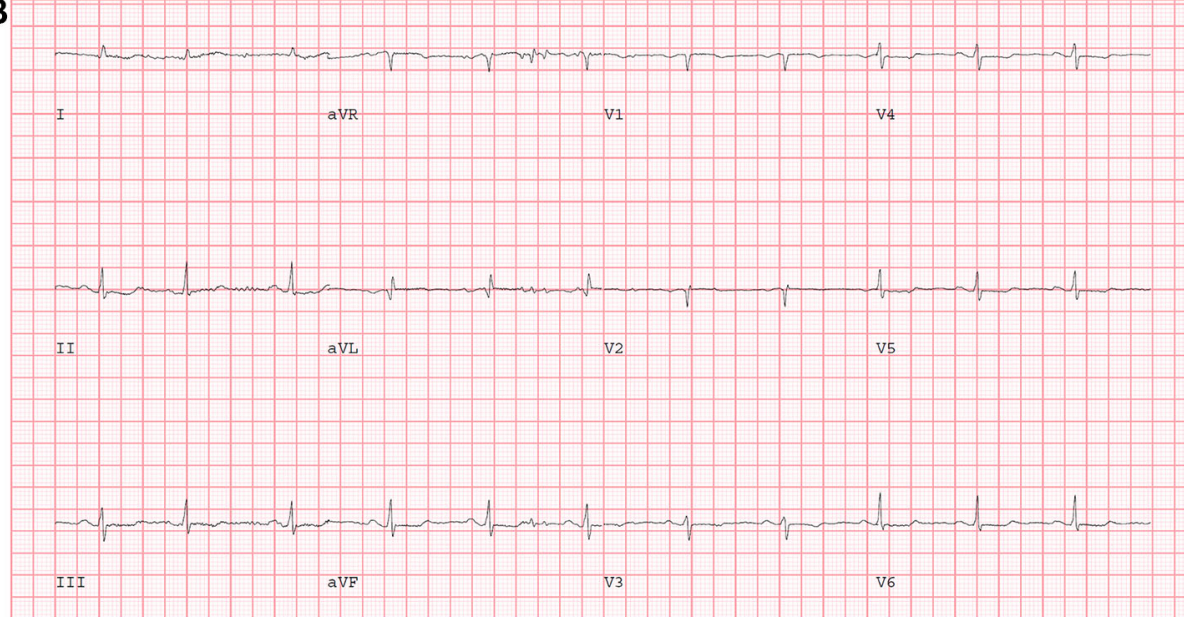

C

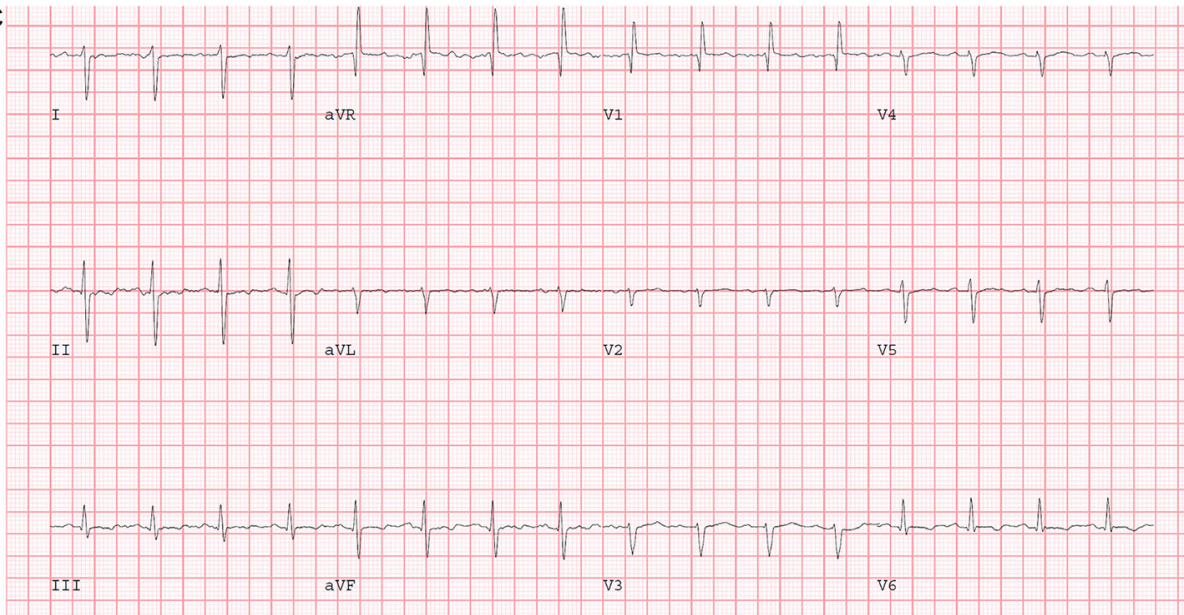

Figure 1. A Representative example of secondary ST/T wave abnormalities of a study patient with left ventricular hypertrophy with strain pattern (all examples courtesy of Rami Doukky, MD, Cook County Health). B Representative example of primary ST wave abnormalities of a study patient. C Representative example of $\mathrm{T}$ wave inversion of a study patient. 
In their evaluation of 1308 patients undergoing dobutamine stress echocardiography (DSE), Pedone et al. found baseline ST/T wave abnormalities to predict MI and cardiac death as well as all-cause mortality independent of the DSE results. ${ }^{2}$ Those with both abnormal ECG and DSE fared the worst. However, an abnormal ECG increased the chance of an adverse outcome even in those with normal DSE.

In a group of patients undergoing both stand-alone exercise testing and coronary angiography Miranda, Lehmann and Froelicher found baseline resting ST depression to predict more severe angiographic CAD and worse infarct-free survival in patients either with and without past myocardial infarction compared to those without baseline ST depression. ${ }^{6}$

In 132 patients undergoing exercise MPI with and without known CAD, Taylor and Beller found baseline ST and $\mathrm{T}$ wave abnormalities to predict a greater frequency of abnormal MPI and when abnormal, greater ischemic extent than in those with a normal baseline ECG. ${ }^{7}$ Elhendy et al. found a group of 246 patients (114 men, 132 women) with baseline ST depression or T wave inversion undergoing dobutamine-atropine MPI to have more defects, fixed or reversible, in the men though not in women. ${ }^{8}$

The study by Khan, Arif, and Doukky ${ }^{1}$ as well as the others discussed above (other than Taylor and Beller $^{7}$ ) excluded patients with known CAD. As reviewed by Miranda et al., numerous studies have found abnormal ST and T wave abnormalities to predict adverse cardiac outcomes in those with known CAD, either among free living in the community or undergoing exercise testing. ${ }^{6}$

\section{CONCLUSION}

Khan et al. have showed us that patients undergoing regadenoson stress MPI with baseline ST and T wave abnormalities have a worse prognosis than those who did not. In their study, $T$ wave inversion did not predict abnormal outcome though it did predict abnormal MPI. As described, previous work has found an abnormal baseline ECG to predict adverse outcome in the general public and in those undergoing exercise testing. These data reinforce that decision making following an MPI study is best performed using a holistic or Bayesian approach. The results of the MPI study include not only MPI perfusion, but substantially more information that is available to the clinician - the patient's symptoms leading up to and during the study, the ECG response to exercise or pharmacologic stress, the patient's co-morbidities including diabetes, renal insufficiency, and past history, the duration of exercise if exercise stress, in addition to the non-perfusion parameters of MPI such as $\mathrm{LV}$ function, transient ischemic dilation, and LV reserve. Khan et al. and earlier researchers add that the baseline ECG includes further incremental information.

As machine learning is applied to MPI, ${ }^{9-11}$ it behooves the research community to continue to add non-perfusion and clinical parameters currently available to clinicians in their post MPI decision making. As machine learning has the potential to integrate even more information than a clinician is capable, it may prove to be a worthy partner.

\section{Disclosures}

Gregory S Thomas acknowledges royalties from Ellestad's Stress Testing, Oxford University Press, 2018 and serving on the Speakers Bureau of Astellas Pharma. Arash Taghav has no disclosures.

\section{References}

1. Khan MS, Arif AW, Doukky R (2020) The prognostic implications of ST-segment and T-wave abnormalities in patients undergoing regadenoson stress SPECT myocardial perfusion imaging. J Nucl Cardiol. https://doi.org/10.1007/s12350-020-023 82-z

2. Pedone C, Elhendy A, van Domburg RT et al (2011) Prognostic significance of baseline ST-T-wave abnormalities in diagnostic stress echocardiography. Coron Artery Dis 22:559-564

3. Daviglus ML, Liao Y, Greenland P et al (1999) Association of nonspecific minor ST-T abnormalities with cardiovascular mortality: The Chicago Western Electric Study. JAMA 281:530-536

4. Dekker JM, Schouten EG, Klootwijk P, Pool J, Kromhout D (1995) ST segment and T wave characteristics as indicators of coronary heart disease risk: The Zutphen Study. J Am Coll Cardiol 25:1321-1326

5. Auer R, Bauer DC, Marques-Vidal P et al (2012) Association of major and minor ECG abnormalities with coronary heart disease events. JAMA 307(14):1497-1505

6. Miranda CP, Lehmann K, Froelicher VF (1991) Correlation between resting ST segment changes, exercise testing, coronary angiography, and long term prognosis. Am Heart J 122:1617

7. Taylor AJ, Beller GA (1994) Correlation of resting electrocardiographic ST-T wave abnormalities with exercise thallium-201 stress testing in patients with known or suspected coronary artery disease. Am J Cardiol 74:211-215

8. Elhendy A, van Domburg RT, Bax JJ, Roelandt JR (1999) Gender differences in the relation between ST-T-wave abnormalities at baseline electrocardiogram and stress myocardial perfusion abnormalities in patients with suspected coronary artery disease. Am J Cardiol 84:865-869

9. Arsanjani R, Xu Y, Dey D et al (2013) Improved accuracy of myocardial perfusion SPECT for detection of coronary artery disease by machine learning in a large population. J Nucl Cardiol 20:553-562 
10. Alonso DH, Wernick MN, Yang Y, Germano G, Berman DS, Slomka P (2019) Prediction of cardiac death after adenosine myocardial perfusion SPECT based on machine learning. J Nucl Cardiol 26:1746-1754

11. Lee BC, Baskaran L, van Rosendael R et al (2019) Development and validation of a SPECT 3D convolutional neural network for diagnosis of vascular territory-specific ischemia: The multicenter
International CREDENCE Trial (abstract). J Nucl Cardiol 26:1452-1496

Publisher's Note Springer Nature remains neutral with regard to jurisdictional claims in published maps and institutional affiliations. 